\title{
Exciting Magnetic Dipole Mode of Split-Ring Plasmonic Nano-Resonator by Photonic Crystal Nanocavity
}

\author{
Yingke Ji ${ }^{1}$, Binbin Wang ${ }^{1}$, Liang Fang ${ }^{1}$, Qiang Zhao ${ }^{2, *}$, Fajun Xiao ${ }^{1}$ and Xuetao Gan ${ }^{1, *}$ \\ 1 Key Laboratory of Light Field Manipulation and Information Acquisition, Ministry of Industry and \\ Information Technology, and Shaanxi Key Laboratory of Optical Information Technology, School of Physical \\ Science and Technology, Northwestern Polytechnical University, Xi'an 710129, China; \\ jiyingke@mail.nwpu.edu.cn (Y.J.); wangbinbin@nwpu.edu.cn (B.W.); fangliang@nwpu.edu.cn (L.F.); \\ fjxiao@nwpu.edu.cn (F.X.) \\ 2 Qian Xuesen Laboratory of Space Technology, China Academy of Space Technology, Beijing 100094, China \\ * Correspondence: zhaoqiang@qxslab.cn (Q.Z.); xuetaogan@nwpu.edu.cn (X.G.)
}

check for updates

Citation: Ji, Y.; Wang, B.; Fang, L.; Zhao, Q.; Xiao, F.; Gan, X. Exciting Magnetic Dipole Mode of Split-Ring Plasmonic Nano-Resonator by Photonic Crystal Nanocavity. Materials 2021, 14, 7330. https:// doi.org/10.3390/ma14237330

Academic Editors: Meng Su, Shengfei Feng, Tianrui Zhai and Zhijia $\mathrm{Hu}$

Received: 17 October 2021

Accepted: 19 November 2021

Published: 30 November 2021

Publisher's Note: MDPI stays neutral with regard to jurisdictional claims in published maps and institutional affiliations.

Copyright: (c) 2021 by the authors. Licensee MDPI, Basel, Switzerland. This article is an open access article distributed under the terms and conditions of the Creative Commons Attribution (CC BY) license (https:// creativecommons.org/licenses/by/ $4.0 /)$.

\begin{abstract}
On-chip exciting electric modes in individual plasmonic nanostructures are realized widely; nevertheless, the excitation of their magnetic counterparts is seldom reported. Here, we propose a highly efficient on-chip excitation approach of the magnetic dipole mode of an individual split-ring resonator (SRR) by integrating it onto a photonic crystal nanocavity (PCNC). A high excitation efficiency of up to $58 \%$ is realized through the resonant coupling between the modes of the SRR and PCNC. A further fine adjustment of the excited magnetic dipole mode is demonstrated by tuning the relative position and twist angle between the SRR and PCNC. Finally, a structure with a photonic crystal waveguide side-coupled with the hybrid SRR-PCNC is illustrated, which could excite the magnetic dipole mode with an in-plane coupling geometry and potentially facilitate the future device application. Our result may open a way for developing chip-integrated photonic devices employing a magnetic field component in the optical field.
\end{abstract}

Keywords: magnetic dipole mode; plasmonic nanoresonator; photonic crystal nanocavity

\section{Introduction}

Tailoring lightwaves at the nanoscale has opened up new opportunities for effectively controlling light-matter interaction in the applications of surface-enhanced Raman scattering (SERS) [1], fluorescence enhancement [2], nonlinear enhancement [3], localized heating [4], optical trapping [5], and chip-integrated passive [6] and active [7] devices. Plasmonic nanostructures, including metallic nanoparticles, nanoshells, nanorods, nanodimers, bowtie antennas and split-ring resonators (SRRs), provide a route to confining lightwaves to a few nanometers [8]. However, the high-efficient excitation of plasmonic modes in these individual nanostructures is still a challenge, which requires strict mode matches in the parameters of polarization, spatial distribution, wavelength, and wavevector. Conventional free-space excitation technology through an objective lens is inefficient due to the orders of magnitude difference between the spot size of focused light and the cross-section of nanostructures. Moreover, the bulky objective lens is undesirable in on-chip integration applications.

The hybrid plasmonic-photonic structures, the combination of plasmonic nanostructures with photonic waveguides and cavities, provide an approach for the effective excitation of plasmonic modes via the near-field coupling. This also shows many attractive performances on the chip-integrated platform. For example, gold nanostructures were placed on photonic crystal to achieve a hybrid plasmonic-photonic cavity with a high quality $(Q)$ factor $[9,10]$, which enables ultra-compact lasers [11], high-throughput biosensors [12], huge electric field enhancement [13], and highly efficient SERS tips [14]. A plasmonic nanodimer was integrated with a microdisk to support chiral emission [15]. Individual plasmonic nanoparticles were combined with a photonic ridge waveguide for 
sensitive sensing [16], with a photonic micro-ring resonator for the highly efficient excitation of a plasmonic nano-resonator mode [17]. Nevertheless, all of them only related to the electric modes of those plasmonic nanostructures; a CMOS-compatible on-chip excitation approach of their magnetic mode is still missing.

As is well known, the photonic crystal (PhC) can be also used for the design of the slotted PhC waveguides and PhC cavity with ultra-high Q and ultra-small Vm [18-26]. Here, the high photon locality of the gold nanostructure and high $Q$ of PhC are combined in an optimal way to propose a robust, reliable, alignment-free, and CMOS-compatible approach to effectively excite the magnetic dipole (MD) mode of an individual SRR by integrating it on a silicon planar photonic crystal nanocavity (PCNC). Metal SRR, as a special plasmonic nanostructure, is widely used in metamaterials as a basic MD due to its magnetic dipole-like mode [27-29]. Here, SRR is chosen as the metal nanostructure to be integrated on the PCNC surface because it has a similar field distribution profile as that of the PCNC, as demonstrated below. The strong polarization dependence of SRR also enables us to realize light field manipulation from near- to far-field. In addition, the ultra-small mode volume together with strong magnetic field enhancement of the PCNC provides a desirable platform for exciting the magnetic mode of the individual SRR. The mode simulation of the hybrid SRR-PCNC indicates the two nano-resonators have very strong mode coupling with an efficiency exceeding $58 \%$. The excited MD mode can be controlled through the gap width of SRR: the relative position and angle between PCNC and SRR. We further illustrate a structure of a photonic crystal waveguide side-coupled with the hybrid SRR-PCNC structure, which could excite the MD mode with an in-plane coupling geometry and potentially facilitate the future device application. The proposed hybrid SRR-PCNC structure opens an avenue to develop magnetic mode-based devices, such as magnetic sensors [30], magnetic nonlinearity [31], and magneto-optic modulation. Furthermore, field enhancement between the SRR-PCNC gap layer promises the improved performance of silicon integrated electro-optic modulators, photodetectors, and sensors.

\section{Resonant Characteristics of Individual SRR and PCNC}

To analyze and understand the coupling between SRR and PCNC, their resonant characteristics are first studied through scattering spectra of individual SRR and PCNC as well as their magnetic field distribution.

Figure 1a shows the schematic of the employed U-shaped gold SRR with outer lengths of $L_{1}=250 \mathrm{~nm}$ and $L_{2}=210 \mathrm{~nm}$, inner length of $g_{y}=125 \mathrm{~nm}$, and gap width $g_{x}=125 \mathrm{~nm}$. The thickness of gold SRR is set as $50 \mathrm{~nm}$. These parameters are optimized to obtain an MD mode of the SRR around the telecom band. The resonant mode of the SRR is simulated by the three-dimensional finite element method (COMSOL Multiphysics). The SRR is set in the air surrounding. For the accuracy of the calculations, the maximum grid size of the model is not larger than one sixth of the light wavelength. The outer boundaries are set as a perfect match layer (PML) for eliminating the interference of the boundary reflection.

With a dipole source excitation located in the center of the SRR, the scattering spectrum of the individual SRR shows two resonant peaks, as shown in Figure 1b, which are the electric quadrupole mode (EQ) and MD mode at the shorter and longer resonant wavelengths, respectively [32]. Compared with dielectric nanocavity, the SRR nanocavity has a lower $Q$ of 5.1 and 3.2 for the EQ and MD mode when the $g_{x}=125 \mathrm{~nm}$, and their value of $Q$ will decrease further as the SRR size increases. Both resonant peaks have a large linewidth and low $Q$ factor due to the strong radiation of dipolar modes and the Ohmic loss from metal, but this in turn facilitates its coupling with the integrated PCNC, as discussed below. The electric fields of the MD mode at the wavelength of $1430 \mathrm{~nm}$ are strongly localized and confined at the ends of SRR's two arms, as shown in Figure 1c1,c2. The induced circular electrical current indicated by white arrows give rise to a typical MD dipole resonance in the gap region of SRR. As shown in Figure 1c3,c4, the magnetic field $H_{z}$ component is concentrated perfectly in the center of SRR, which reveals that the direction of dipole moment is perpendicular to the current plane, i.e., the SRR plane. 

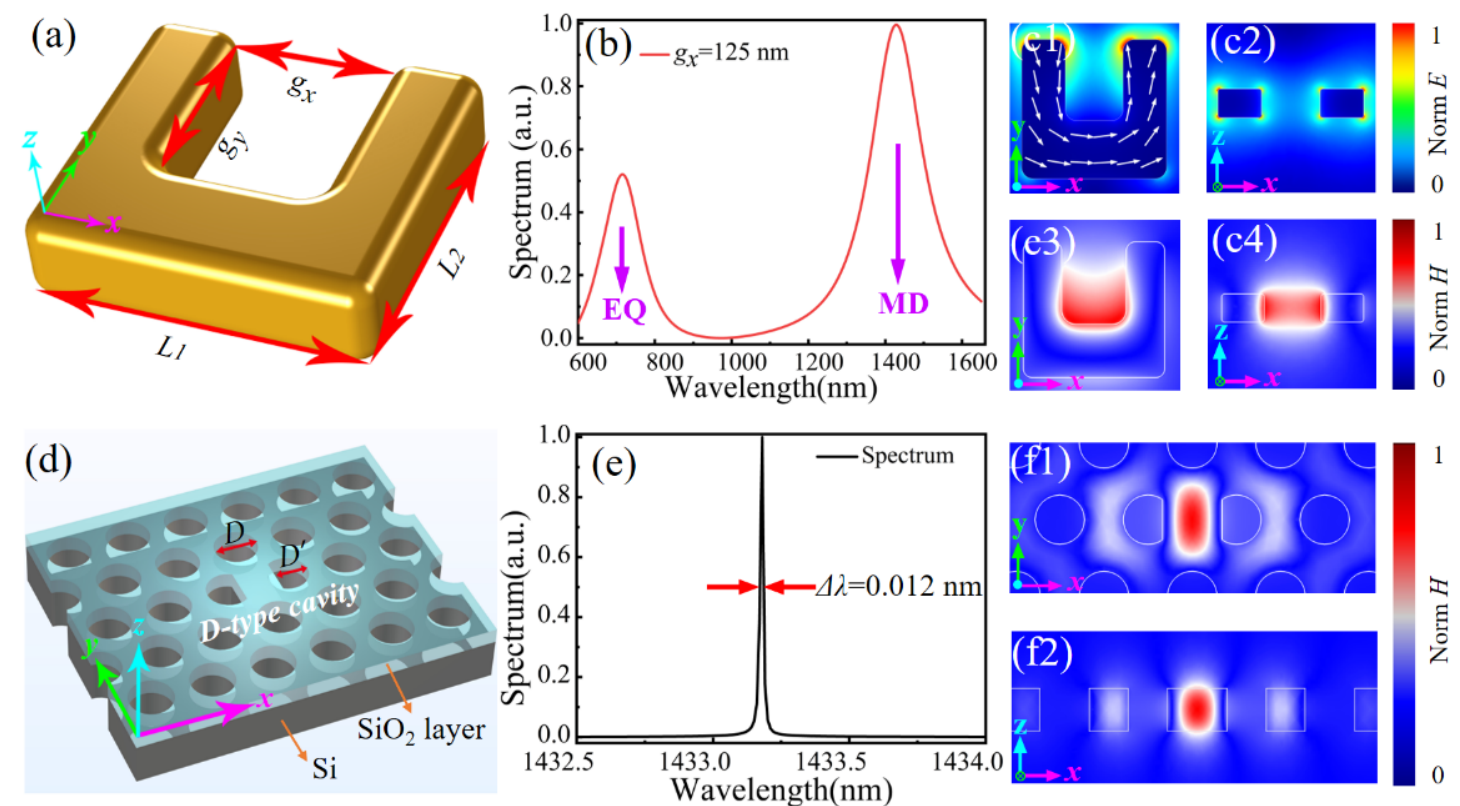

Figure 1. (a) Schematic of a gold SRR with defined geometric parameters. (b) Calculated scattering spectrum of an individual SRR showing EQ and MD resonant modes. (c1,c2) Electric field and (c3,c4) magnetic field distributions of SRR at the resonant wavelength of $1430 \mathrm{~nm}$. (d) Schematic of the D-type PCNC with D' $=0.8 \mathrm{D}$. (e) Calculated scattering spectrum of the D-type PCNC. (f1,f2) Magnetic field distributions of D-type PCNC at $x-y$ and $x-z$ cross-sections, respectively.

The PCNC employed to integrate the SRR is a D-type, which has an ultra-small mode volume and a high $Q$ factor, as shown in Figure 1d. The cavity is formed by cutting two inner adjacent air holes into a $\mathrm{D}$ shape with a width of $\mathrm{D}^{\prime}=0.8 \mathrm{D}$ in a hexagonal photonic crystal lattice, where D is the optimized diameter of the air hole [33]. The PCNC is constructed in a $220 \mathrm{~nm}$ thick silicon slab, and the lattice constant of the air holes is $454 \mathrm{~nm}$, while the air-hole radius is $127 \mathrm{~nm}$. PCNCs have been widely studied and designed with different defect structures. To evaluate the employee D-shape PCNC, we show a brief comparison of their $Q$ and $V_{\mathrm{m}}$ values in Table 1. The optimized point-defect PCNC has the highest $Q=5.02 \times 10^{6}$ and air slot PCNC presents a minimum of $V_{\mathrm{m}}=0.042(\lambda / n)^{3}[34,35]$. The employed D-shape cavity formed by cutting two air holes exhibits a $Q$ factor of $10^{5}$ and $V_{\mathrm{m}}$ of $0.329(\lambda / n)^{3}$. Compared with other PC nanocavities, the D-shape cavity has single and concentrated magnetic field distribution, which can more efficiently realize the coupling with the magnetic mode of SRR. Here, because a thin $\mathrm{SiO}_{2}$ cladding layer with the air holes being the same as those on the silicon slab is required to integrate the Au SRR on the D-shape PCNC, the asymmetric top and bottom cladding layers cause the decrease in $Q$ and increase in $V_{\mathrm{m}}$.

Table 1. Comparison of different PCNCs.

\begin{tabular}{cccc}
\hline Structure & Quality Factor $(\mathbf{Q})$ & Mode Volume $\left(V_{\mathbf{m}}\right)$ & Ref. \\
\hline Heterostructure PCNC & $4 \times 10^{4}$ & $1.46(\lambda / n)^{3}$ & {$[34]$} \\
Air slot heterostructure PCNC & $2.6 \times 10^{4}$ & $0.042(\lambda / n)^{3}$ & {$[35]$} \\
Optimized point-defect PCNC & $5.02 \times 10^{6}$ & $0.6(\lambda / n)^{3}$ & {$[23]$} \\
D-shape PCNC & $2.005 \times 10^{5}$ & $0.329(\lambda / n)^{3}$ & {$[33]$} \\
\hline
\end{tabular}

In the proposed hybrid SRR-PCNC structure, there is a $100 \mathrm{~nm}$ thick $\mathrm{SiO}_{2}$ spacing layer between them. Consistent with the future device preparation by depositing a $\mathrm{SiO}_{2}$ spacing layer on top of the $\mathrm{PCNC}$, the air holes will not be filled by $\mathrm{SiO}_{2}$. Hence, the fundamental resonant mode of the D-type PCNC covered with a $100 \mathrm{~nm}$ thick $\mathrm{SiO}_{2}$ layer is considered here. The obtained resonant wavelength is $1433 \mathrm{~nm}$, as indicated in the scattering spectrum shown in Figure 1e. The resonant peak shows a very narrow linewidth 
of $0.012 \mathrm{~nm}$, corresponding to a $Q$ factor of $1.15 \times 10^{5}$. Figure $1 \mathrm{f} 1$,f2 display the magnetic field distribution of the fundamental resonant mode of PCNC at the $x-y$ and $x-z$ crosssections, which is strongly localized in the defect region between the two D-type air holes and penetrates the outside of D-type holes. Well-confined mode distribution represents an ultra-small mode volume of $V_{\mathrm{m}}=0.0342(\lambda / n)^{3}$. The ultrasmall mode volume together with strong magnetic field enhancement of D-type PCNC promise a desirable platform for exciting the magnetic mode of the individual SRRs through the mode-matching technique.

Figure 2 shows the calculated permeability of gold SRR in the infrared frequency region. Since the operation of the SRR is based on the $L C$ resonant circuit (a popular design for the magnetic "atoms" is to mimic a usual $L C$ circuit) coupled with a magnetic field, we determine the frequency dispersion of conduction characteristics of metal to fully describe the SRR's behavior with the help of COMSOL. As shown in Figure 2a, the real part of permeability of SRR is retrieved from the computed scattering and S-para parameter [36-38]. In case of $g_{x}=125 \mathrm{~nm}$, there are two regions with an obvious perturbation of $\mu$ caused by the EQ resonance and MD resonance of SRR. With the increase in $g_{x}$, the magnetic resonance of SRR shifts to the longer wavelength. Thus, we just observe one perturbation of the $\mu$ region as $g_{x}=250 \mathrm{~nm}$. To emphasize and understand the relationship between $g_{x}$ and the permeability of SRR, we show the permeability distribution with the changing of $g_{x}$ in Figure 2b. Compared with permeability, the permittivity of SRR presents a bigger range value and a wider jump region with the changing wavelength shown in Figure $2 \mathrm{c}, \mathrm{d}$. Both $\mu$ and $\varepsilon$ shift; the perturbation region shifts to the longer wavelength with the increase in $g_{x}$. These properties can help us understand the perturbation theory of the electromagnetic field in the paper. Compared with the excitation via incidence of plane wave or dipole source, there are more complicated situations when SRR is excited by PCNC. This is because the angle degree and electric polarization cannot be accurately determined. The exact magnitude of permeability and permittivity cannot be derived when SRR is integrated on the PCNC, but the general trend can be inferred from the above results.
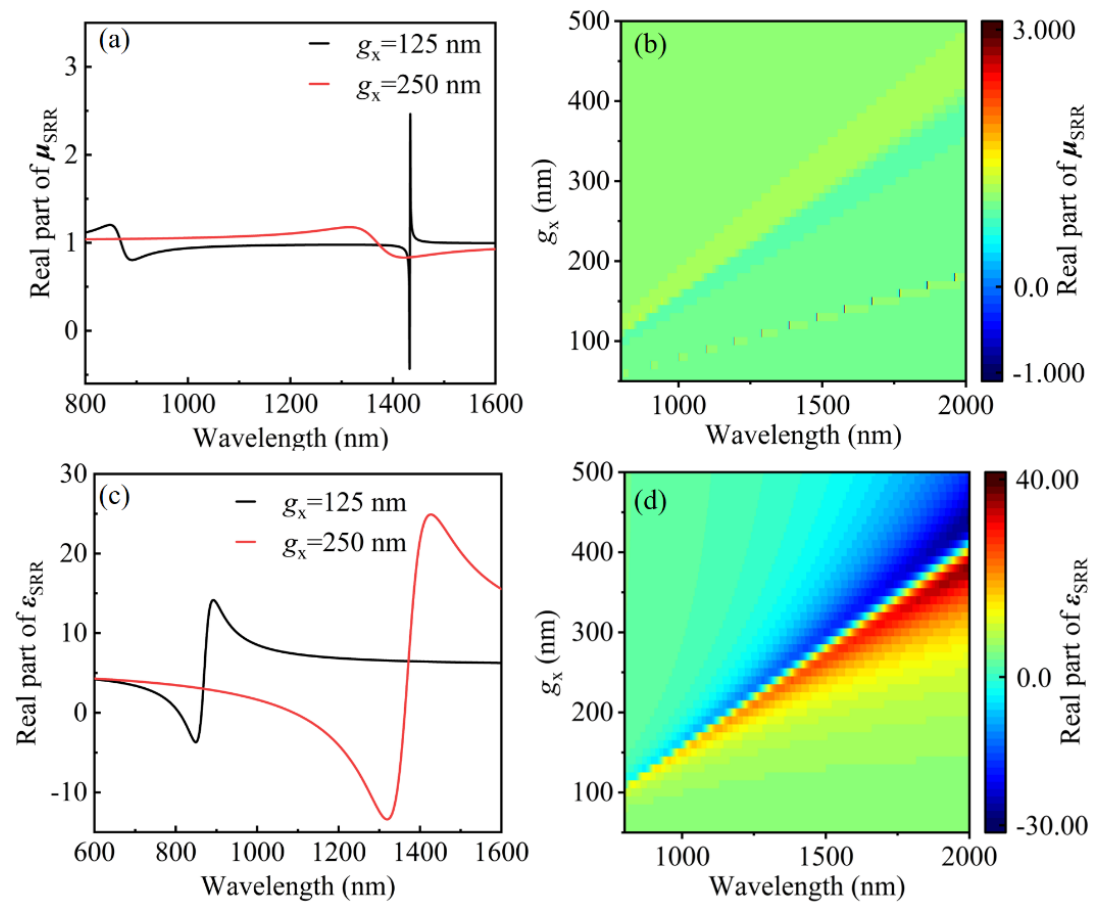

Figure 2. (a) The real part of the retrieved effective permeability $\mu$ around the plasmon resonant of single SRR. (b) The $\mu$ distribution about the $g_{x}$ size of SRR from 50 to $500 \mathrm{~nm}$. (c) The real part of the retrieved effective permittivity $\varepsilon$ around the plasmon resonant of single SRR. (d) The $\varepsilon$ distribution with the $g_{x}$ size of SRR from 50 to $500 \mathrm{~nm}$. 


\section{Magnetic Dipole Mode Excitation: Strong Coupling between SRR and PCNC}

Figure 3a schematically displays the hybrid SRR-PCNC structure, which has a $100 \mathrm{~nm}$ thick $\mathrm{SiO}_{2}$ spacing layer between them. Since the SRR is located at the center of the PCNC, resonant coupling between their modes would be realized. Perturbation of the PCNC resonant mode by the SRR could be observed due to the large effective permittivity and permeability of the resonant modes from the gold SRR. On the other hand, SRR's MD mode would be effectively excited by the near-field of the PCNC mode.
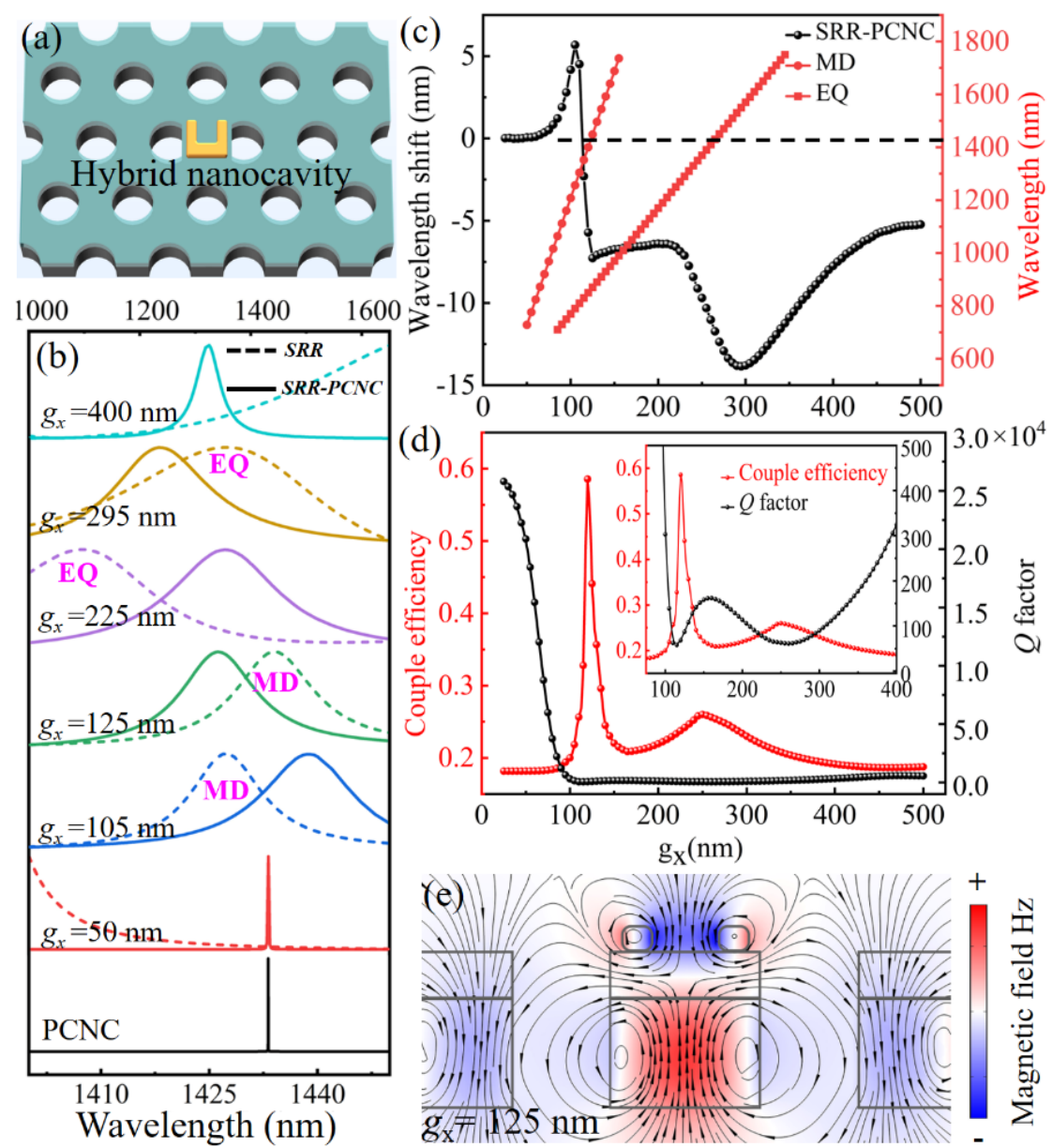

Figure 3. (a) Schematic of the proposed hybrid SRR-PCNC structure. (b) Scattering spectra of individual SRR and hybrid SRR-PCNC structures as a function of the SRR gap width. (c) Resonant wavelength of MD and EQ modes of individual SRR (right), and resonant wavelength shift $\Delta \lambda$ of a hybrid SRR-PCNC structure relative to the resonant wavelength of the bare PCNC as functions of the SRR gap width. (d) Variations of $Q$ factors of hybrid SRR-PCNC structure and coupling efficiency between SRR and PCNC with different SRR gap widths. Inset: Zoomed $Q$ factor at the flat region. (e) Simulated magnetic field distribution of MD mode of hybrid SRR-PCNC structure ( $x-z$ crosssection) with the SRR gap width of $125 \mathrm{~nm}$.

To this end, we first change the gap width $g_{x}$ of SRR to study the coupling effect between SRR and PCNC in view of the fact that $g_{x}$ strongly affects the resonant wavelength of SRR [39]. As indicated in Figure 3b, MD and EQ modes of SRR sequentially appear around the resonant peak of PCNC (at $1433 \mathrm{~nm}$ ) when $g_{x}$ is increased gradually. As a result, the scattering spectra of hybrid SRR-PCNC structures show a dynamic change. As $g_{x}=50 \mathrm{~nm}$, the emission spectrum exhibits a narrow line width, and the resonant wavelength hardly changes. Especially, as the resonance wavelength of the MD or EQ modes of SRR overlap the peak of PCNC, the spectrum of SRR-PCNC presents an obvious blueshift and wider line width when $g_{x}=125$ and $295 \mathrm{~nm}$. 
To explain the variation of the scattering spectra of the hybrid SRR-PCNC structure as a function of the SRR gap width of $g_{x}$, we plot the resonant wavelengths of MD and EQ modes of individual SRR and the resonant wavelength shift $\Delta \lambda$ of the hybrid SRR-PCNC structure relative to the resonant wavelength of PCNC, as shown in Figure 3c. As the SRR gap width $g_{x}$ increases from 50 to $500 \mathrm{~nm}$ gradually, the resonant peaks of the MD mode and EQ mode overlap with the resonant peak of PCNC successively. As a result of their mode couplings, the resonant mode of SRR would induce an effective permeability perturbation $\mu_{S R R}$ and an effective permittivity perturbation $\varepsilon_{S R R}$ to the resonant mode of PCNC, respectively. In turn, this leads to a resonant frequency shift $\Delta \omega$ or $\Delta \lambda$ of PCNC, which is given by a perturbation theory $[40,41]$.

$$
\Delta \omega \sim \frac{\omega_{\mathrm{n}}}{2} \frac{\delta \mu\left|H_{\mathrm{n}, z}\left(\vec{r}_{0}\right)\right|^{2}}{\iiint_{-\infty}^{+\infty} r \vec{H}_{n}^{*} \cdot \vec{H}_{n}(\vec{r}) d V}
$$

where $\omega_{\mathrm{n}}$ is the resonant frequency of PCNC, $\vec{H}_{\mathrm{n}}(\vec{r})$ and $\delta$ and $\mu$ are the unperturbed magnetic field and the magnetic perturbation, respectively. $\vec{r}_{0}$ is the position of SRR. Both the perturbations from permittivity $\varepsilon_{S R R}$ and permeability $\mu_{S R R}$ cause the resonant frequency shift $\Delta \lambda$. The MD mode is getting close to the mode of PCNC at $1433 \mathrm{~nm}$ when $g_{x}$ increases gradually (Figure $1 \mathrm{~b}, \mathrm{c}$ ). This induces a permeability perturbation $\mu_{S R R}>0$ at the wavelength of $1433 \mathrm{~nm}$, as calculated in Figure 2, and hence leads to a red shift of the resonant wavelength of the hybrid SRR-PCNC structure (Figure 3c). As indicated by Equation (1), $\mu_{S R R}$ decreases to 0 when $g_{x}=105 \mathrm{~nm}$, whereas a dramatic decrease in $\Delta \lambda$ is observed in Figure 3c. When the gap width $g_{x}$ changed from 105 to $125 \mathrm{~nm}, \mu_{S R R}$ remains negative. According to Equation (1), the blue shift of the resonant wavelength of the hybrid SRR-PCNC happens in this case. Especially, when the MD resonant wavelength matches the PCNC mode wavelength at $g_{x}=125 \mathrm{~nm}$, a maximum inductive magnetic field in the SRR is obtained, as shown in Figure 3d. When the range of $g_{x}$ changed from 125 to $225 \mathrm{~nm}$, both MD and EQ modes are weak at $1433 \mathrm{~nm}$, and $\Delta \lambda$ is nearly flat. With the further increase in gap width $\left(g_{x}>225 \mathrm{~nm}\right)$, the EQ mode dominates in SRR and takes over the MD mode. In this case, SRR first presents a negative permittivity $\varepsilon_{S R R}$ and gives rise to an extraordinary blue shift of the resonant wavelength of the hybrid SRR-PCNC. The maximum blue shift happens at $g_{x}=295 \mathrm{~nm}$, where $\varepsilon_{S R R}=0$. As the resonant wavelength of the EQ mode moves away from the PCNC mode when $g_{x}>295 \mathrm{~nm}, \varepsilon_{S R R}$ is positive, which causes a redshift of the resonant wavelength of the SRR-PCNC structure.

To find a suitable $g_{x}$ to effectively excite the MD mode of SRR, we plot the coupling efficiency $\eta$ and $Q$ factor of the hybrid SRR-PCNC structure as a function of the gap width $g_{x}$ (Figure 3e). Here, the coupling efficiency $\eta$ is calculated by [42].

$$
\eta=\frac{\iiint_{S R R}\left(\mu_{0}|\overrightarrow{\mathbf{H}}|^{2}+\varepsilon_{0}|\overrightarrow{\mathbf{E}}|^{2}\right) d V}{\iiint_{-\infty}^{+\infty}\left(\mu_{0}|\overrightarrow{\mathbf{H}}|^{2}+\varepsilon_{0}|\overrightarrow{\mathbf{E}}|^{2}\right) d V}
$$

where $\mu_{0}$ and $\varepsilon_{0}$ are the vacuum permeability and vacuum permittivity, $\vec{H}$ and $\vec{E}$ represent the magnetic and electric, respectively.

According to the black curve in Figure 3b, the individual PCNC has a high $Q$ factor. Integrating an SRR on PCNC introduces a perturbation to the resonant mode of PCNC, leading to a reduction of $Q$ factor with the increase in $g_{x}$. For a small $g_{x}$, the MD resonant peak of the SRR is far away from the resonant peak of PCNC, as shown by the red curves in Figure 3b, which indicates weak coupling between SRR and PCNC; then, a high $Q$ factor inherits from PCNC (Figure 3e). The increased $g_{x}$ moves the MD resonant peak of SRR close to that of the PCNC (Figure $3 b$ ) and hence enhances perturbation to the resonant 
mode of PCNC, which brings a drastic drop of the $Q$ factor of the hybrid SRR-PCNC structure (Figure 3d). The $Q$ factor nearly keeps flat if $g_{x}>100 \mathrm{~nm}$, which is caused by the dramatic growth of loss of the SRR. If we enlarge the view of Figure $3 d$ in the inset, we can clearly observe a fluctuation of $Q$ values. A minimum $Q$ factor appears at $g_{x}=105 \mathrm{~nm}$ (inset of Figure 3d), which indicates the strongest perturbation of the SRR to PCNC. Close to this position, the maximum coupling efficiency of $58 \%$ is obtained at $g_{x}=125 \mathrm{~nm}$, where the MD resonant peak of the SRR has an overlap with the resonant peak of PCNC. The MD mode of the SRR is effectively excited at $g_{x}=125 \mathrm{~nm}$ (Figure 3e). Another minimum $Q$ factor point appears at $g_{x}=260 \mathrm{~nm}$ and the maximum coupling efficiency of $26 \%$ is obtained at $g_{x}=250 \mathrm{~nm}$ (inset of Figure 3d). In this case, the EQ resonant peak of the SRR is close to the resonant peak of PCNC instead of the MD peak (Figure 3b). Hence, the EQ mode of the SRR is effectively excited at $g_{x}=250 \mathrm{~nm}$.

To evaluate the intrinsic properties of the hybrid SRR-PCNC and the strength of coupling between the MD mode of the SRR and the resonant mode of the PCNC, the mode volume $V_{\mathrm{m}}$ and figure of merit $Q / V_{\mathrm{m}}$ are calculated in Figure $4 \mathrm{a}, \mathrm{b}$. The values of $V_{\mathrm{m}}$ have two dips when the gap width of SRR increases. This is because the MD mode and EQ mode of SRR are excited by the PCNC mode as $g_{x}=125 \mathrm{~nm}$ and $250 \mathrm{~nm}$, which cause the strong confinement of photons. Meanwhile, the values of $Q / V_{\mathrm{m}}$ also have peaks as $g_{x}=125 \mathrm{~nm}$ and $250 \mathrm{~nm}$, which indicate the higher Purcell effect of the SRR-PCNC. The overall declined trend of $Q / V_{\mathrm{m}}$ as the $g_{x}$ increased is because of the rapid decrease in $Q$ factors due to the increased metal loss with the larger SRR. The comparison of coupling efficiency in the hybrid plasmonic-photonic structures is shown in Table 2. Note that in our proposed SRR-PCNC structure, the MD mode is successfully excited with a high coupling efficiency. The coupling efficiency of the hybrid SRR-PCNC can be improved by optimizing the size and position of the SRR to increase the overlapping between the PCNC mode and MD mode of the SRR.
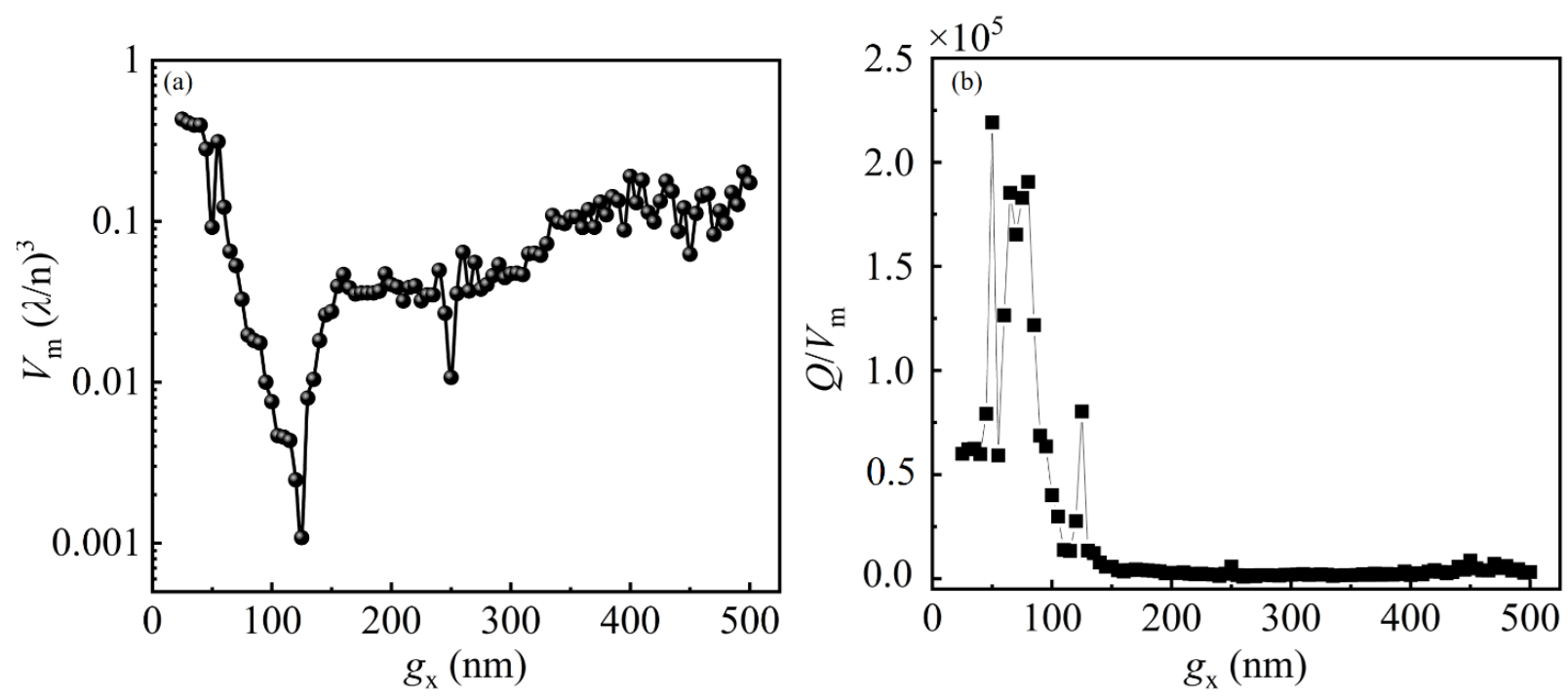

Figure 4. (a) The calculated $V_{\mathrm{m}}$ as a function of $g_{x}$. (b) The simulated $Q / V_{\mathrm{m}}$ ratio as the function of $g_{x}$.

Table 2. Comparison of coupling efficiency of the hybrid structure.

\begin{tabular}{cccc}
\hline Structure & $\eta$ & $\begin{array}{c}\text { Excitation of MD } \\
\text { (Yes or No) }\end{array}$ & Ref. \\
\hline Gold spheres dimer on PhC cavity & $40 \%$ & No & {$[9]$} \\
Gold bowtie antenna on PhC cavity & $62 \%$ & No & {$[13]$} \\
Gold particle on waveguide & $9.7 \%$ & No & {$[16]$} \\
Gold particle on ring resonator & $78 \%$ & No & {$[17]$} \\
Nanowires on PCNC & $1.9 \%$ & No & {$[42]$} \\
Gold SRR on PCNC & $58 \%$ & Yes & - \\
\hline
\end{tabular}




\section{Effect of Relative Position and Angle between SRR and PCNC}

Adjusting the gap width of SRR offers a versatile method to control the excitation of the MD mode. However, it introduces an undesirable EQ mode. In this section, we present the fine controlling of the excitation of the MD mode through the relative position and angle between the SRR and PCNC. The SRR with the fixed $g_{x}=125 \mathrm{~nm}$ is employed to provide the MD mode overlapping with the mode of PCNC.

Figure 5a schematically shows the horizontal distance of SRR, $x_{p}$, relative to the center of the PCNC denoted by the arrows. The scattering spectra of the hybrid SRR-PCNC structures exhibit periodic red-shifted and blue-shifted resonant peaks as the SRR moves from left to right gradually, as shown in Figure 5b. It needs to be pointed out that the line width of the resonant spectrum indicates the couple between the SRR and PCNC and loss of the hybrid SRR-PCNC structure. For example, as the $x_{p}= \pm a$, the narrow line width reveals the weak coupling and lower loss due to the scattering and absorption of the SRR. Resonant wavelength shifts with respect to the resonant wavelength of the bare PCNC as the SRR moves are shown in Figure 5c. The change of wavelength red shift and blue shift could be attributed to the weak and strong coupling between the MD mode of the SRR and the PCNC mode with different magnetic field intensity, which is governed by Equation (1). The value of $V_{\mathrm{m}}$ as the function of the SRR position is also shown with a red line. The minimum value of $V_{\mathrm{m}}$ indicates the most confinement of photons determined by the coupling strength of the hybrid system. In addition, the different linewidths in Figure $5 \mathrm{~b}$ indicate the different coupling efficiency between the SRR and PCNC as well, which is extracted as the $Q$ factors of the hybrid SRR-PCNC structure, as shown in Figure $5 \mathrm{~d}$. Both the coupling efficiency and $Q$ factor indicate the strong interaction between the SRR and PCNC when the SRR locates in the position where the PCNC mode has the maximum magnetic field at $x_{p}=0, \pm a$, and weak interaction when $x_{p}= \pm a / 2$ where the magnetic field is close to 0 .
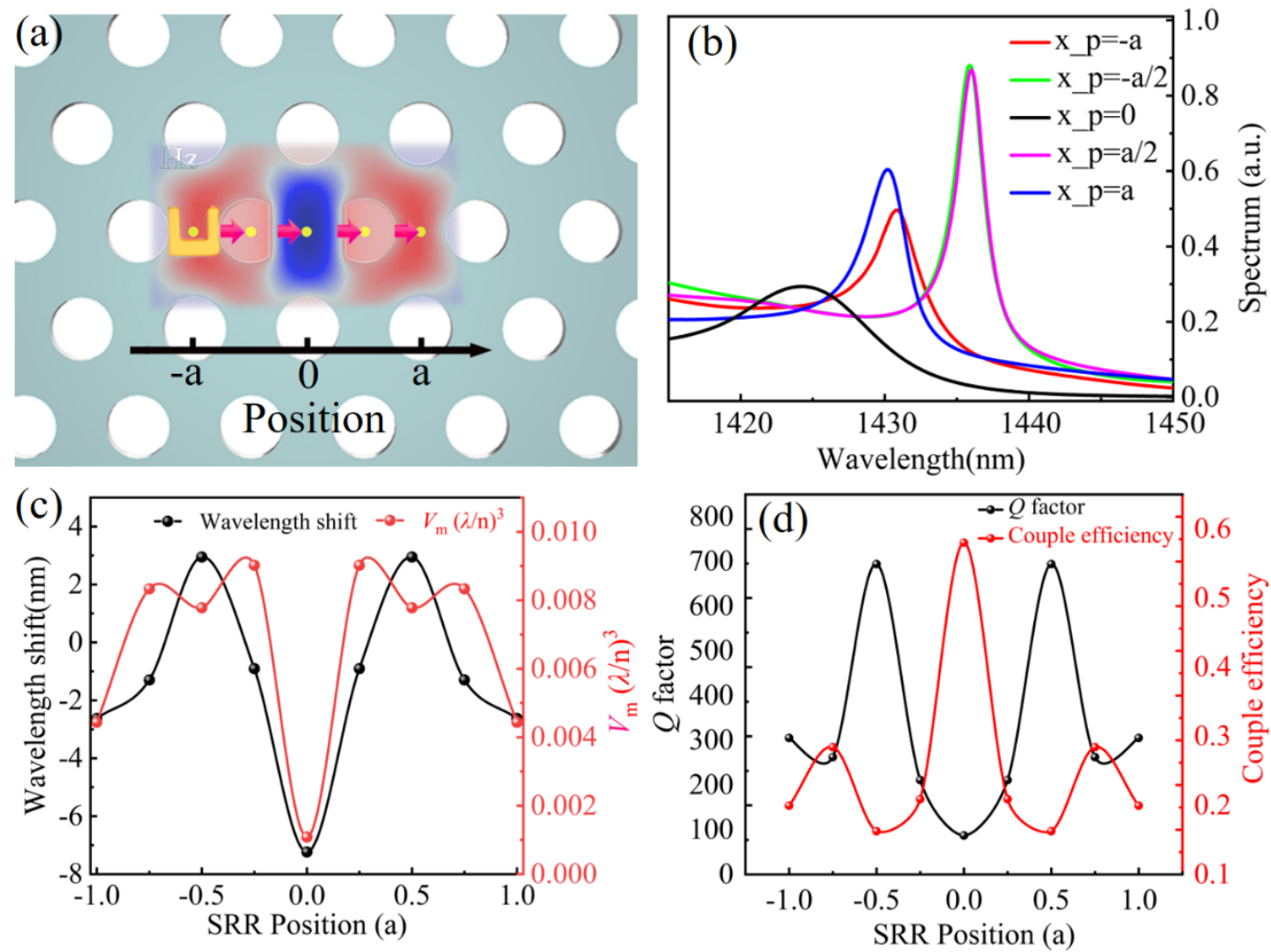

Figure 5. (a) Schematic for the changing relative position between the SRR and D-type PCNC. The magnetic field distribution of the mode in PCNC at $1433 \mathrm{~nm}$ is superposed onto the structure. (b) Scattering spectra of the MD mode of the hybrid SRR-PCNC structure with different relative positions. (c) Resonant wavelength shift and value of $V_{\mathrm{m}}$ under different relative positions. (d) $Q$ factor and coupling efficiency as a function of the relative position. 
Tuning the SRR direction with different relative angle $\theta$ with respect to the PCNC is another method to finely adjust the MD mode of the SRR, as schematically shown in Figure 6a. An obvious red shift and sharpening of the resonant peak are observed from the scattering spectra of the hybrid SRR-PCNC structure when the relative angle is changed from $0^{\circ}$ to $90^{\circ}$, as shown in Figure $6 \mathrm{~b}$. These results can be interpreted by the change of coupling efficiency between SRR and PCNC. When the relative angle changes from $0^{\circ}$ to $90^{\circ}$, the magnetic field overlapping between the SRR and PCNC will decrease gradually. This means that the coupling efficiency between the SRR and PCNC will be reduced, corresponding to a decrease in the magnetic response and loss. Then, there is a bigger permeability of SRR according to Figure 2, which leads to the sharpening of the peak and red shift of the resonant wavelength.
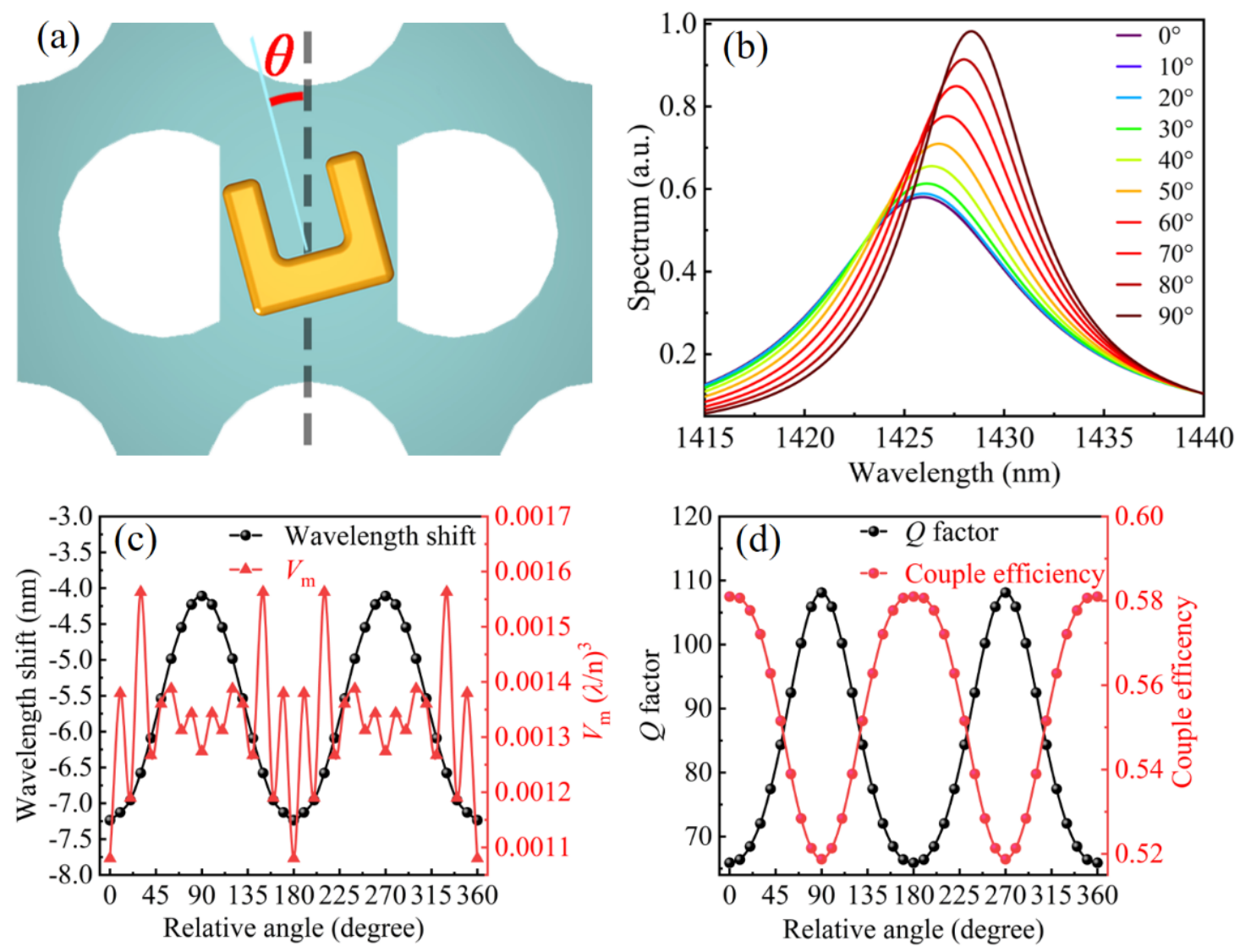

Figure 6. (a) Schematic of the turning relative angle $\theta$ between SRR and PCNC. (b) Scattering spectra of hybrid SRR-PCNC structure with different relative angle from $0^{\circ}$ to $90^{\circ}$. (c) Resonant wavelength shift $\Delta \lambda$ and value of $V_{\mathrm{m}}$ as a function of $\theta$. (d) Coupling efficiency $\eta$ and $Q$ factor as functions of $\theta$.

The resonant wavelength shift $\Delta \lambda$ remains negative due to the negative permeability of the SRR and exhibits periodic variation similar to the sinusoidal function of $\theta$ (Figure 6c). When $\theta=0^{\circ}, 180^{\circ}$, and $360^{\circ}$, the resonant wavelength has a maximum blue shift, indicating the strongest coupling between the SRR and PCNC. When $\theta=90^{\circ}$ and $270^{\circ}$, the resonant wavelength shows the minimum blue shift, showing the weak coupling between the SRR and PCNC. The maximum blueshift corresponds to the minimum of $V_{\mathrm{m}}$, which predicts the strongest confinement of photons as the most coupling between the SRR and PCNC. This is confirmed by the coupling efficiency and $Q$ factor curves in Figure 6d. The coupling efficiency ( $Q$ factor) gradually weakens (increases) when $\theta$ increases from $0^{\circ}$ to $90^{\circ}$, and it enhances (decreases) from $90^{\circ}$ to $0^{\circ}$. 


\section{All Integrated On-Chip Excitation of Magnetic Dipole Mode of SRR}

To facilitate the future device applications of the near-field excited MD mode of SRR in the hybrid SRR-PCNC structure, we further propose to side-couple the SRR-PCNC structure with a photonic crystal waveguide, enabling the in-plane accessing geometry.

Figure 7a is the schematic to excite the MD mode of the SRR in the integrated architecture. The cavity mode of the PCNC is excited through a photonic crystal line-defect waveguide, which then excites the MD mode of the SRR. By optimizing the mode overlap between the PCNC and the photonic crystal waveguide, which have a separation with three rows of air holes, their evanescent field coupling efficiency could be as high as 78\% [42]; as shown by the $x-y$ cross-section mode profile in Figure $7 \mathrm{~b}$, the resonant mode of $\mathrm{D}$-type PCNC is effectively excited by the photonic crystal waveguide, immediately following effective excitation of the MD mode of the SRR. As shown in the cross-section view of Figure $7 \mathrm{c}, \mathrm{d}$, the magnetic field of the resonant mode in the PCNC can effectively couple into that of the SRR. As calculated from Equation (2), the excitation efficiency of the SRR MD mode by the PCNC mode is $58 \%$. Hence, the total excitation efficiency of the SRR MD mode is estimated as $45 \%$ from the side-coupled photonic crystal waveguide. Finally, we also calculated the transmission spectra of SRR-PCNC and PCNC, respectively, as shown in the inset of Figure 7d. Corresponding to the resonant characteristics of the PCNC and SRR-PCNC, a broader and blue-shifted resonant dip is obtained from the SRR-PCNC compared with that of the PCNC. These results support the all-integrated on-chip excitation of the MD mode of the SRR, paving the way to applications in chip-integrated magnetic-optic devices.
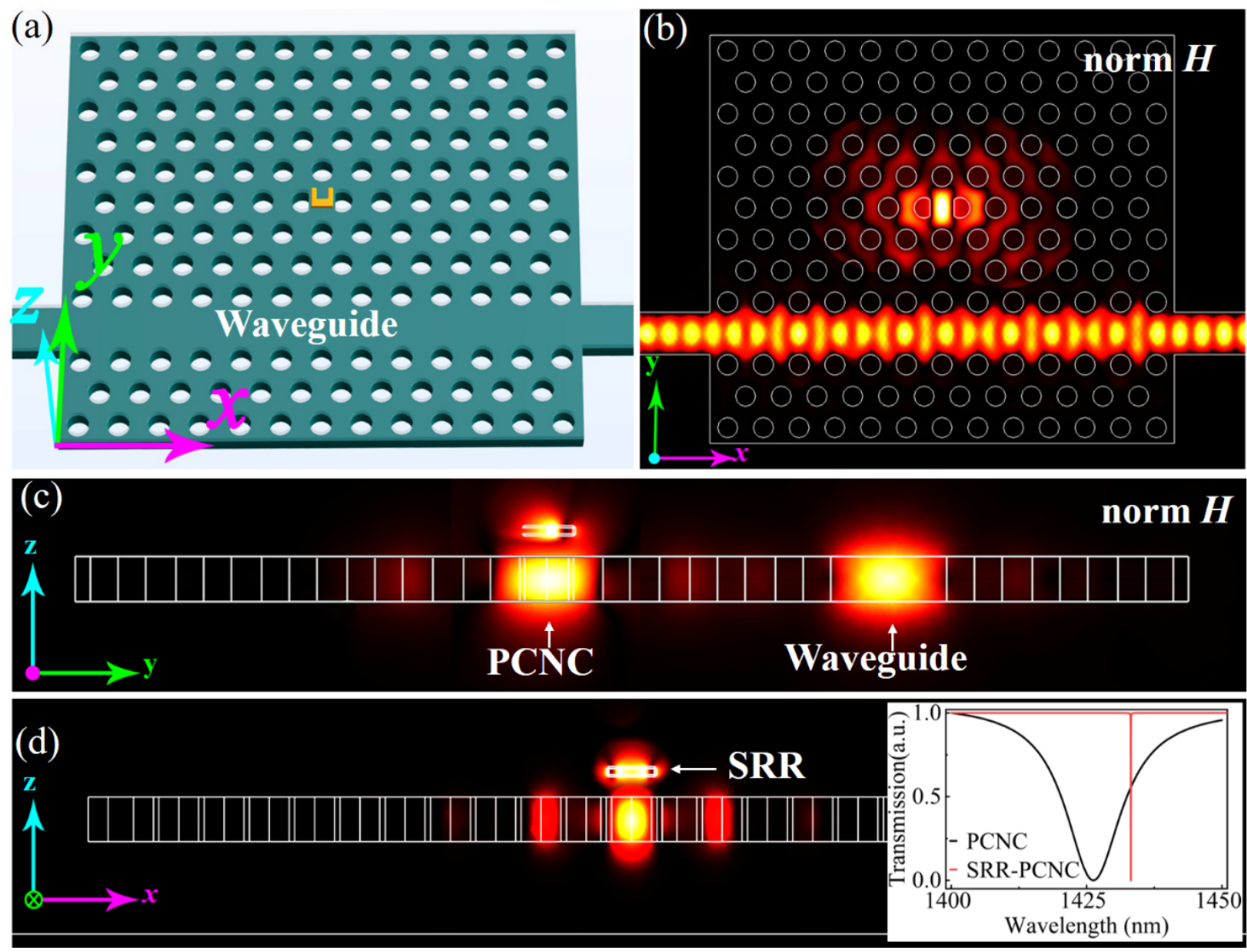

Figure 7. (a) Schematic of all integrated on-chip excitation of the MD mode in the SRR by side-coupling the hybrid SRR-PCNC with a photonic crystal waveguide. (b-d) Simulated magnetic field distribution of the proposed configuration in (a) from the views of the $x-y$ cross-section through the center of the silicon slab (b), $y-z$ cross-section (c), and $x-z$ cross-section through the center of the PCNC (d), respectively. The inset is the calculated transmission spectrum of the SRR-PCNC and PCNC side-coupled by a waveguide. 


\section{Conclusions}

In conclusion, we have demonstrated an excitation approach of the MD mode of the SRR by integrating it onto a silicon PCNC, which eliminates the use of a bulky objective lens and significantly improves the efficiency. This benefits from the strong near-field mode coupling between the SRR and PCNC. The coupling mechanism in the hybrid SRR-PCNC structure is numerically and theoretically illustrated with the help of an electromagnetic perturbation theory and finite element method (FEM). A coupling efficiency between the SRR MD mode and PCNC mode as high as 58\% indicates the highly efficient on-chip excitation of the SRR MD mode. A fine adjustment of the excitation of the MD mode is presented through the varied relative position and angle between the SRR and PCNC. This result shows the intensity distribution of the near-field in the PCNC and reveals the potential application in magnetic imaging. An all-integrated excitation configuration of the MD mode is finally described by side coupling a photonic crystal waveguide with the PCNC. Due to the limitation of experimental conditions, the above results cannot be verified by experiments for the time being. Therefore, we use the finite difference time domain (FDTD) technique to verify the simulation and find that the results of the two algorithms are consistent. Our approach may open the way for the on-chip applications of a magnetic field component in the optical field, such as a magnetic sensor [30], magnetooptic modulator [31,43-45], second harmonic manipulation [46], all-optical switch [47], microwave sensor [48], plasmonic nanolasers, and spacer [49-51].

Author Contributions: Conceptualization, X.G.; Funding acquisition, X.G.; Investigation, Y.J. and X.G.; Methodology, Y.J. and L.F.; Writing—original draft, Y.J.; Writing—review \& editing, B.W., Q.Z., F.X. and X.G. All authors have read and agreed to the published version of the manuscript.

Funding: This research was funded by the Key Research and Development Program 2017YFA0303800, in part by the National Natural Science Foundation 91950119, 11634010, 61775183, and 61905196, in part by the Key Research and Development Program in Shaanxi Province of China 2020JZ-10, in part by the Fundamental Research Funds for the Central Universities 310201911cx032 and 3102019JC008.

Institutional Review Board Statement: Not applicable.

Informed Consent Statement: Not applicable.

Conflicts of Interest: The authors declare no conflict of interest.

\section{References}

1. Maier, S.A. Plasmonic field enhancement and SERS in the effective mode volume picture. Opt. Express 2006, 14, 1957. [CrossRef]

2. Tam, F.; Goodrich, G.P.; Johnson, B.R.; Halas, N.J. Plasmonic enhancement of molecular fluorescence. Nano Lett. $2007,7,496-501$. [CrossRef] [PubMed]

3. Kauranen, M.; Zayats, A.V. Nonlinear plasmonics. Nat. Photonics 2012, 6, 737-748. [CrossRef]

4. Jauffred, L.; Samadi, A.; Klingberg, H.; Bendix, P.; Oddershede, L.B. Plasmonic Heating of Nanostructures. Chem. Rev. 2019, 119, 8087-8130. [CrossRef] [PubMed]

5. Maragò, O.M.; Jones, P.H.; Gucciardi, P.G.; Volpe, G.; Ferrari, A.C. Optical trapping and manipulation of nanostructures. Nat. Nanotechnol. 2013, 8, 807-819. [CrossRef] [PubMed]

6. Wang, B.; Blaize, S.; Salas-Montiel, R. Nanoscale plasmonic TM-pass polarizer integrated on silicon photonics. Nanoscale 2019, 11, 20685-20692. [CrossRef] [PubMed]

7. Wang, B.; Blaize, S.; Seok, J.; Kim, S.; Yang, H.; Salas-Montie, R. Plasmonic-Based Subwavelength Graphene-on-hBN Modulator on Silicon Photonics. IEEE J. Sel. Top. Quantum Electron. 2019, 25, 1-6. [CrossRef]

8. Maier, S.A. Plasmonics: Fundamentals and Applications; Springer: New York, NY, USA, 2007.

9. Barth, M.; Schietinger, S.; Fischer, S.; Becker, J.; Nusse, N.; Aichele, T.; Lochel, B.; Sönnichsen, C.; Benson, O. Nanoassembled plasmonic-photonic hybrid cavity for tailored light-matter coupling. Nano Lett. 2010, 10, 891-895. [CrossRef]

10. Zhang, H.; Liu, Y.C.; Liu, Y.; Wang, C.; Zhang, N.; Lu, C. Hybrid photonic-plasmonic nano-cavity with ultra-high Q/V. Opt. Lett. 2020, 45, 4794-4797. [CrossRef]

11. Zhang, T.; Callard1, S.; Jamois, C.; Chevalier, C.; Feng, D.; Belarouci, A. Plasmonic-photonic crystal coupled nanolaser. Nanotechnology 2014, 25, 315201. [CrossRef]

12. Mossayebi, M.; Parini, A.; Wright, A.J.; Somekh, M.G.; Bellanca, G.; Larkins, E. Hybrid photonic-plasmonic platform for highthroughput single-molecule studies. Opt. Mater. Express 2019, 9, 2511-2522. [CrossRef] 
13. Eter, A.E.; Grosjean, T.; Viktorovitch, P.; Letartre, X.; Benyattou, T.; Baida, F.I. Huge light-enhancement by coupling a bowtie nano-antenna's plasmonic resonance to a photonic crystal mode. Opt. Express 2014, 22, 14464. [CrossRef] [PubMed]

14. Angelis, F.D.; Das, G.; Candeloro, P.; Patrini, M.; Galli, M.; Bek, A.; Lazzarino, M.; Maksymov, I.; Liberale, C.; Andreani, L.C.; et al. Nanoscale chemical mapping using three-dimensional adiabatic compression of surface plasmon polaritons. Nat. Nanotechnol. 2010, 5, 67-72. [CrossRef]

15. Cognée, K.G.; Doeleman, H.M.; Lalanne, P.; Koenderink, A.F. Cooperative interactions between nano-antennas in a high-Q cavity for unidirectional light sources. Light Sci. Appl. 2019, 8, 115. [CrossRef]

16. Chamanzar, M.; Xia, Z.; Yegnanarayanan, S.; Adibi, A. Hybrid integrated plasmonic-photonic waveguides for on-chip localized surface plasmon resonance (LSPR) sensing and spectroscopy. Opt. Express 2013, 21, 32086. [CrossRef] [PubMed]

17. Chamanzar, M.; Adibi, A. Hybrid nanoplasmonic-photonic resonators for efficient coupling of light to single plasmonic nanoresonators. Opt. Express 2011, 19, 22292. [CrossRef]

18. Akahane, Y.; Asano, T.; Song, B.S.; Noda, S. Fine-tuned high-Q photonic-crystal nanocavity. Opt. Express 2005, 13, 1202-1214. [CrossRef] [PubMed]

19. Nomura, M.; Tanabe, K.; Iwamoto, S.; Arakawa, Y. High-Q design of semiconductor-based ultrasmall photonic crystal nanocavity. Opt. Express 2010, 18, 8144-8150. [CrossRef]

20. Kassa-Baghdouche, L.; Boumaza, T.; Bouchemat, M. Optimization of Q-factor in nonlinear planar photonic crystal nanocavity incorporating hybrid silicon/polymer material. Phys. Scr. 2015, 90, 065504. [CrossRef]

21. Kassa-Baghdouche, L.; Boumaza, T.; Bouchemat, M. Optical properties of point-defect nanocavity implemented in planar photonic crystal with various low refractive index cladding materials. Appl. Phys. B 2015, 121, 297-305. [CrossRef]

22. Kassa-Baghdouche, L.; Boumaz, T.; Cassan, E.; Bouchemat, M. Enhancement of Q-factor in SiN-based planar photonic crystal L3 nanocavity for integrated photonics in the visible-wavelength range. Optik 2015, 126, 3467-3471. [CrossRef]

23. Nakamura, T.; Takahashi, Y.; Tanaka, Y.; Asano, T.; Noda, S. Improvement in the quality factors for photonic crystal nanocavities via visualization of the leaky components. Opt. Express 2016, 24, 9541-9549. [CrossRef] [PubMed]

24. Kassa-Baghdouche, L.; Cassan, E. Mid-infrared refractive index sensing using optimized slotted photonic crystal waveguides. Photonics Nanostruct.-Fundam. Appl. 2018, 28, 32. [CrossRef]

25. Kassa-Baghdouche, L. High-sensitivity spectroscopic gas sensor using optimized H1 photonic crystal microcavities. J. Opt. Soc. Am. B 2020, 37, A277-A284. [CrossRef]

26. Kassa-Baghdouche, L. Optical properties of a point-defect nanocavity-based elliptical-hole photonic crystal for mid-infrared liquid sensing. Phys. Scr. 2020, 95, 015502. [CrossRef]

27. Smith, D.R.; Padilla, W.J.; Vier, D.C.; Nemat-Nasser, S.C.; Schultz, S. Composite medium with simultaneously negative permeability and permittivity. Phys. Rev. Lett. 2000, 84, 4184-4187. [CrossRef]

28. Yen, T.J.; Padilla, W.J.; Fang, N.; Vier, D.C.; Smith, D.R.; Pendry, J.B.; Basov, D.N.; Zhang, X. Terahertz Magnetic Response from Artificial Materials. Science 2004, 303, 1494-1496. [CrossRef]

29. Linden, S.; Enkrich, C.; Wegener, M.; Zhou, J.; Koschny, T.; Soukoulis, C.M. Magnetic response of metamaterials at 100 terahertz. Science 2004, 306, 1351-1353. [CrossRef]

30. Vourc'h, E.; Joubert, P.-Y. Analytical and numerical analyses of a current sensor using non linear effects in a flexible magnetic transducer. Prog. Electromagn. Res. 2009, 99, 323-338. [CrossRef]

31. Klein, M.W.; Enkrich, C.; Wegener, M.; Linden, S. Second-harmonic generation from magnetic metamaterials. Science 2006, 313, 502-504. [CrossRef]

32. Liu, N.; Guo, H.; Fu, L.; Schweizer, H.; Kaiser, S.; Giessen, H. Electromagnetic resonances in single and double split-ring resonator metamaterials in the near infrared spectral region. Phys. Status Solidi B 2007, 244, 1251-1255. [CrossRef]

33. Fang, L.; Gan, X.; Zhao, J. High-Q factor photonic crystal cavities with cut air holes. Chin. Opt. Lett. 2020, 18, 86-92.

34. Sünner, T.; Stichel, T.; Kwon, S.-H.; Schlereth, T.W.; Höfling, S.; Kamp, M.; Forchel, A. Photonic crystal cavity based gas sensor. Appl. Phys. Lett. 2008, 92, 26112. [CrossRef]

35. Jágerská, J.; Zhang, H.; Diao, Z.; Thomas, N.L.; Houdré, R. Refractive index sensing with an air-slot photonic crystal nanocavity. Opt. Lett. 2010, 35, 2523-2525. [CrossRef] [PubMed]

36. Smith, D.R.; Vier, D.C.; Koschny, T.; Soukoulis, C.M. Electromagnetic parameter retrieval from inhomogeneous metamaterials. Phys. Rev. E 2005, 71, 036617. [CrossRef] [PubMed]

37. Tanaka, T.; Ishikawa, A.; Kawata, S. Negative Permeability of Single-ring Split Ring Resonator in the Visible Light Frequency Region. Mater. Res. Soc. Symp. Proc. 2006, 919, 0919-J03-08. [CrossRef]

38. Linden, S.; Enkrich, C.; Dolling, G.; Klein, M.W.; Zhou, J.; Koschny, T.; Soukoulis, C.M.; Burger, S.; Schmidt, F.; Wegener, M. Photonic Metamaterials: Magnetism at Optical Frequencies. IEEE J. Sel. Top. Quantum Electron. 2006, 12, 1097-1105. [CrossRef]

39. Rockstuhl, C.; Zentgraf, T.; Guo, H.; Liu, N.; Etrich, C.; Loa, I.; Syassen, K.; Kuhl, J.; Lederer, F.; Giessen, H. Resonances of split-ring resonator metamaterials in the near infrared. Appl. Phys. B Lasers Opt. 2006, 84, 219-227. [CrossRef]

40. Vignolini, S.; Intonti, F.; Riboli, F.; Balet, L.; Li, L.H.; Francardi, M.; Gerardino, A.; Fiore, A.; Wiersma, D.S.; Gurioli, M. Magnetic imaging in photonic crystal microcavities. Phys. Rev. Lett. 2010, 105, 123902. [CrossRef]

41. Lalouat, L.; Cluzel, B.; Velha, P.; Picard, E.; Peyrade, D.; Hugonin, J.P.; Lalanne, P.; Hadji, E.; de Forne, F. Near-field interactions between a subwavelength tip and a small-volume photonic-crystal nanocavity. Phys. Rev. B 2007, 76, 041102. [CrossRef] 
42. Yuan, Q.; Fang, L.; Zhao, Q.; Wang, Y.; Mao, B.; Khayrudinov, V.; Lipsanen, H.; Sun, Z.; Zhao, J.; Gan, X. Mode couplings of a semiconductor nanowire scanning across a photonic crystal nanocavity. Chin. Opt. Lett. 2019, 17, 80-85. [CrossRef]

43. Joannopoulos, J.D.; Johnson, S.G.; Winn, J.N.; Meade, R.D. Photonic Crystals: Molding the Flow of Light, 2nd ed.; Princeton University Press: Princeton, NJ, USA, 1995.

44. Liang, D.; Bowers, J.E. Recent Progress in Heterogeneous III-V-on-Silicon Photonic Integration. Light Adv. Manuf. 2021, 2, 5. [CrossRef]

45. Hadad, Y.; Davoyan, A.R.; Engheta, N.; Steinberg, B.Z. Extreme and Quantized Magneto-optics with Graphene Meta-atoms and Metasurfaces. ACS Photonics 2014, 1, 1068-1073. [CrossRef]

46. Tsai, W.-Y.; Chung, T.L.; Hsiao, H.-H.; Chen, J.; Lin, R.; Wu, P.; Sun, G.; Wang, C.; Misawa, H.; Tsai, D.P. Second Harmonic Light Manipulation with Vertical Split Ring Resonators. Adv. Mater. 2019, 31, 1806479. [CrossRef] [PubMed]

47. Kondo, Y.; Murai, T.; Shoji, Y.; Mizumoto, T. All-Optical Switch by Light-to-Heat Conversion in Metal Deposited Si Ring Resonator. IEEE Photonics Technol. Lett. 2020, 32, 807-810. [CrossRef]

48. Wu, J.; Yang, D.; Huang, X.; Li, Y.; Xia, Y. The design and experiment of a novel microwave gas sensor loaded with metamaterials. Phys. Lett. A 2021, 389, 127080. [CrossRef]

49. Ning, C.Z. Semiconductor nanolasers and the size-Energy efficiency challenge: A review. Adv. Photonics 2019, 1, 014002. [CrossRef]

50. Oulton, R.F.; Sorger, V.J.; Zentgraf, T.; Ma, R.-M.; Gladden, C.; Dai, L.; Bartal, G.; Zhang, X. Plasmon lasers at deep subwavelength scale. Nature 2009, 461, 629-632. [CrossRef] [PubMed]

51. Ding, Z.; Huang, Z.; Chen, Y.; Mou, C.; Lu, Y.; Xua, F. All-fiber ultrafast laser generating gigahertz-rate pulses based on a hybrid plasmonic microfiber resonator. Adv. Photonics 2020, 2, 026002. [CrossRef] 